\title{
Does an "Overactive to Underactive Bladder Transition" Phenomenon Exist in a Rat Lumbar Spinal Canal Stenosis Model?
}

\author{
Noritoshi Sekido', Jun Kida², Daisuke Wakamatsu², Hiroki Okada², Hidekazu Matsuya² \\ ${ }^{1}$ Department of Urology, School of Medicine, Faculty of Medicine, Toho University Medical Center Ohashi \\ Hospital, Tokyo, Japan \\ ${ }^{2}$ Group2 Discovery Research Laboratories, Ono Pharmaceutical Co., Ltd., Osaka, Japan \\ Email: nsekido@med.toho-u.ac.jp
}

Received 11 April 2015; accepted 6 May 2015; published 8 May 2015

Copyright (C) 2015 by authors and Scientific Research Publishing Inc.

This work is licensed under the Creative Commons Attribution International License (CC BY).

http://creativecommons.org/licenses/by/4.0/

(c) ()

\section{Abstract}

Purpose: To investigate the association between the maximum cystometric capacity (MCC) and other cystometric parameters in lumbar canal stenosis (LCS) rats. Material and Methods: One small hole was drilled at the fifth lumbar vertebral arch (Sham), and a rectangular piece of silicone rubber was then placed in the epidural space (LCS) of Wister rats. Two weeks after surgery, awake cystometry was performed. LCS rats were divided into three groups: Group A (n = 5, MCC < 0.87 $\mathrm{mL})$, Group B (n = 13, MCC $0.87-1.81 \mathrm{~mL})$, and Group C $(\mathrm{n}=14, \mathrm{MCC}>1.81 \mathrm{~mL})$. Cystometric parameters were investigated in sham and LCS groups. Results: MCC did not significantly correlate to the frequency of non-voiding contractions (NVCs), voided volume (VV), or maximum intravesical pressure during voiding (Pmax), but significantly positively correlated to postvoid residual urine volume (PVR) and residual urine rate (RUR) (Spearman's correlation coefficients $(\rho)=0.8973$ ( $p<$ $0.0001)$ and $0.4915(p=0.0068)$, respectively). Compared with the sham rats, LCS rats in each group revealed significantly smaller VV, larger RUR, and lower Pmax. On the other hand, among LCS rats, VV, RUR, and Pmax were not significantly different. The frequency of NVCs in each LCS group was not significantly different from that in sham-operated rats (Tukey-Kramer's HSD test). However, a Jonckheere-Terpstra trend test revealed a significant trend toward higher NVCs in the order of sham, Groups C, B, and A $(p=0.036)$. Conclusions: LCS rats showed the same degree of detrusor underactivity regardless of MCC. NVCs did not significantly increase in LCS rats with decreased MCC, but the trend toward higher NVCs with smaller MCC was significant.

\section{Keywords}

Underactive Bladder, Detrusor Underactivity, Lumbar Canal Stenosis, Animal Model, 


\section{Overactive Bladder}

\section{Introduction}

A recent clinical study demonstrated that underactive bladder (UAB) could be preceded by overactive bladder (OAB) which is regarded as a compensatory mechanism for decreased shortening velocity [1]. Early detrusor underactivity (DU) is frequently accompanied by detrusor overactivity (DO, 68\%), while advanced DU is not (18\%). In a basic experiment, Nomiya et al. investigated bladder function in an atherosclerosis rat model that was induced by endothelial injury of the internal iliac artery with a high cholesterol diet [2]. These rats manifested an OAB phenotype in a metabolic cage study and on awake cystometry. However, in vivo muscle strip studies showed impaired detrusor contractility. Based on these studies, some researchers proposed the existence of an “OAB to UAB transition” [3]-[6].

Recently, we reported a rat model of lumbar canal stenosis (LCS) as a UAB model [7] [8]. This model was created by insertion of a small piece of silicone rubber into the L5 to L6 epidural space to compress the cauda equina, and it reveals features of infrasacral neuropathic bladder (NB), namely, impairment of voiding efficiency and a decrease in detrusor contractility. Unexpectedly, maximum cystometric capacity (MCC, mL) in some LCS rats became smaller than that in sham rats, which is why MCC in LCS rats $(1.58 \pm 0.68 \mathrm{~mL}$, mean \pm SEM) was not significantly different from that in sham rats $(1.34 \pm 0.23 \mathrm{~mL})$ [7]. In clinical settings, it was reported that DO was noted in 29\% of LCS patients [9]. This phenomenon seemed to be caused by irritation of the sacral roots, ischemic disturbances of the conus medullaris, or decentralization of the parasympathetic ganglia situated within the bladder wall [9] [10]. In this study we investigated whether MCC was increased in LCS rats. Our hypotheses were: 1) voiding parameters in LCS rats with normal or decreased MCC would be better; 2) the frequency of non-voiding contractions (NVCs, /min) would be increased in LCS rats with decreasing MCC.

\section{Materials and Methods}

All experiments were approved by the Animal Experimental Committee of Ono Pharmaceutical Co., Ltd. This study further explored awake cystometric findings from a previous study [7]. The procedures of model preparation and the awake cystometric study are briefly described as below.

Model preparation: Female Wistar rats (180 - 190 g, CLEA, Inc., n = 32) were anesthetized with pentobarbital (40 mg/kg, i.p.: Schering-Plough Animal Health), and the fifth and sixth lumbar vertebral arches were exposed. One small hole $(1.5 \mathrm{~mm}$ in diameter) was drilled at the fifth vertebral arch. A rectangular piece $(3.5 \times 5.0 \times 0.5$ $\mathrm{mm}$ ) of silicone rubber (Yamanaka Chemical Ind., Ltd.) was then inserted into the L5 - L6 epidural space. LCS rats underwent bladder expression at least twice a day. An identical surgical procedure was performed on control sham-operated animals $(n=10)$ except that the silicone rubber was not placed in the epidural space. An aliquot of urine was collected before each experiment and examined microscopically for leukocytes in order to rule out significant urinary tract infection (UTI). As shown in the previous study [7], UTI was not identified on urinalysis at CMG, and histological studies of the bladder specimens showed no obvious qualitative difference between sham and LCS rats. Also, there was no significant renal impairment on blood chemistry at the time of CMG. The cystometric study was performed 14 days after surgery in the present study.

Awake cystometry (CMG): Cystometric investigations were performed without any anesthesia in a restraining cage (W $80 \mathrm{~mm} \times$ L $300 \mathrm{~mm} \times$ H 150 mm, Yamanaka Chemical Ind., Ltd.) 3 d after suprapubic bladder catheter implantation in 10 sham-operated rats and 32 LCS rats. Micturition volumes were measured by a fluid collector connected to a force displacement transducer (NSI-022, BrainScience Idea Co., Ltd.) placed under the urethral orifice. The bladder catheter was connected via a T-tube to a pressure transducer (DX-100, Nihon Kohden Corp.) and a microinjection pump (Terufusion Syringe Pump TE-311, Terumo Corp.). Room temperature saline was infused into the bladder at a rate of $2 \mathrm{~mL} / \mathrm{h}$. All rats were given $60 \mathrm{~min}$ to acclimate to test conditions before starting CMGs. Residual urine volume was measured after each micturition reflex by evacuating the bladder through the cystometric catheter. Cystometric parameters were acquired three times for each rat, and data from the second and third measurements were analyzed. The cystometric parameters investigated were MCC; frequency of NVCs per min, which is characterized by fluctuations in intravesical pressure larger than $3.04 \mathrm{mmHg}$ 
without voiding [11]; voided volume (VV, $\mathrm{mL})$; postvoid residual urine volume (PVR, $\mathrm{mL})$; and maximum bladder pressure during micturition (Pmax, mmHg). The residual urine rate (RUR, \%) was calculated by the equation $(\mathrm{PVR} \div(\mathrm{VV}+\mathrm{PVR})) \times 100$. Cystometric data are presented as the mean $\pm \mathrm{SEM}$.

The correlation of MCC or the frequency of NVCs with other cystometric parameters was assessed with Spearman's correlation coefficient $(\rho)$. We considered $|\rho| \geq 0.7$ as being strongly correlated, $0.7>|\rho| \geq 0.5$ as being correlated, $0.5>|\rho| \geq 0.3$ as being weakly correlated, and $0.3>|\rho|$ as being uncorrelated. Then, the LCS rats were divided into three groups: Group A $(n=5$, MCC $<0.87 \mathrm{~mL}$, Figure 1$)$, Group B ( $n=13$, MCC 0.87 $1.81 \mathrm{~mL}$, Figure 1), and Group C $(\mathrm{n}=14$, MCC $>1.81 \mathrm{~mL}$, Figure 1). These ranges corresponded to less than $-2 \mathrm{SD},-2 \mathrm{SD}$ to $+2 \mathrm{SD}$, and more than +2SD of MCC in sham rats, respectively. First, Cystometric parameters including NVCs, VV, PVR, RUR, and Pmax, were investigated in sham and LCS groups using an analysis of variance (ANOVA) test. When the results from ANOVA revealed a statistical significance, then, Tukey-Kramer's HSD test was performed as a post-hoc test. Finally, we examined whether a Jonckheere-Terpstra trend test revealed a significant trend toward higher NVCs in the order of sham, Groups C, B, and A. Analysis was performed by JMP 9.0.2 (SAS Institute Inc.). For all statistical tests, $\mathrm{p}<0.05$ was considered significant.

\section{Results}

\subsection{MCC vs. VV, PVR, RUR, and Pmax}

MCC was not significantly correlated to VV, or Pmax, but was significantly positively correlated to PVR and RUR (Spearman's correlation coefficients $(\rho)=0.8973(\mathrm{p}<0.0001)$ and $0.4915(\mathrm{p}=0.0068)$, respectively, Table 1, Figure 2). In sham and each LCS group ANOVA revealed statistical differences $(p<0.0001)$ in VV, PVR, RUR, and Pmax. Compared with the sham rats, LCS rats in each group revealed significantly smaller VV, larger RUR, and lower Pmax. On the other hand, among LCS rats VV, RUR, and Omax were not significantly different (Table 2, Figure 3). VV in LCS rats ranged from $0.062 \pm 0.086 \mathrm{~mL}$ to $0.141 \pm 0.058 \mathrm{~mL}$. PVR in Group A $(0.50 \pm 0.14 \mathrm{~mL})$ was significantly smaller than in Group B $(1.3 \pm 0.087 \mathrm{~mL})$ or C $(1.92 \pm 0.094 \mathrm{~mL})$,

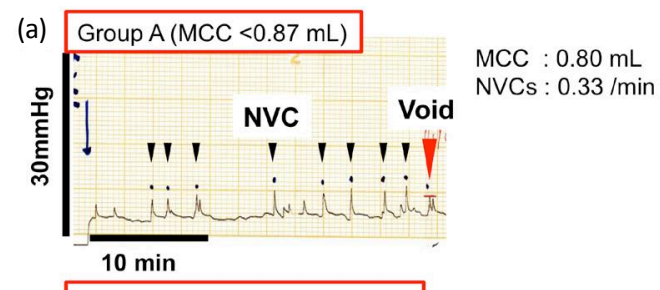

(b). Group B (MCC $0.87-1.81 \mathrm{~mL})$

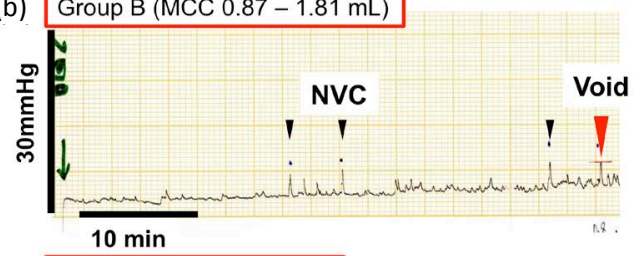

(c) Group B (MCC >1.81 mL)

MCC : $2.40 \mathrm{~mL}$ NVCs : $0.00 / \mathrm{min}$

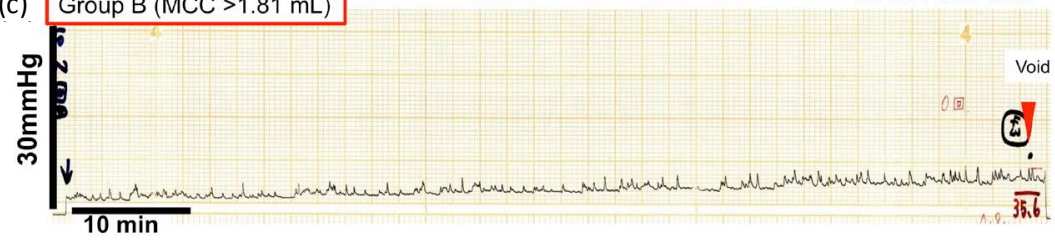

Figure 1. Representative cystometric traces of each group. (a) Group A: the range of MCC corresponds to less than -2SD of MCC in sham rats. Maximum cystometric capacity (MCC), $0.80 \mathrm{~mL}$; the frequency of non-voiding contractions (NVCs), $0.33 / \mathrm{min}$; (b) Group B: the range of MCC corresponds to -2SD to +2SD of MCC in sham rats. MCC, $1.25 \mathrm{~mL}$, NVCs, $0.08 / \mathrm{min}$; (c) Group C: the range of MCC corresponds to more than +2SD of MCC in sham rats. MCC, $2.50 \mathrm{~mL}$; NVCs, $0.00 / \mathrm{min}$. "Void" indicates the time when the first micturition occurs. 
Table 1. Spearman correlation coefficients $(\rho)$ of maximum cystometric capacity (MCC) and frequency of non-voiding contractions (NVCs) with other cystometric parameters.

\begin{tabular}{|c|c|c|c|c|c|}
\hline & NVCs (/min) & $\mathrm{VV}(\mathrm{mL})$ & PVR (mL) & RUR (\%) & Pmax (mmHg) \\
\hline \multicolumn{6}{|c|}{ MCC (mL) } \\
\hline$\rho$ & -0.1412 & 0.0421 & 0.8973 & 0.4915 & 0.1873 \\
\hline $\mathrm{p}$ & 0.4409 & 0.8283 & $<0.0001$ & 0.0068 & 0.3305 \\
\hline \multicolumn{6}{|c|}{ NVCs (/min) } \\
\hline$\rho$ & & 0.1065 & -0.2581 & -0.1993 & -0.0341 \\
\hline $\mathrm{p}$ & & 0.5824 & 0.1765 & 0.3001 & 0.8606 \\
\hline
\end{tabular}

VV, voided volume; PVR, postvoid residual urine volume; RUR, residual urine rate $(=(\mathrm{PVR} \div(\mathrm{VV}+\mathrm{PVR})) \times 100)$; Pmax, maximum bladder pressure during micturition.

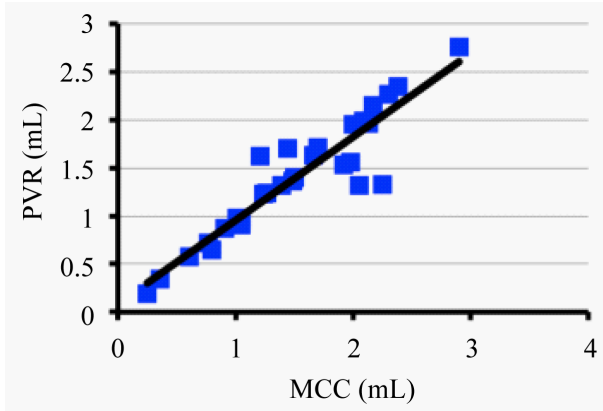

(a)

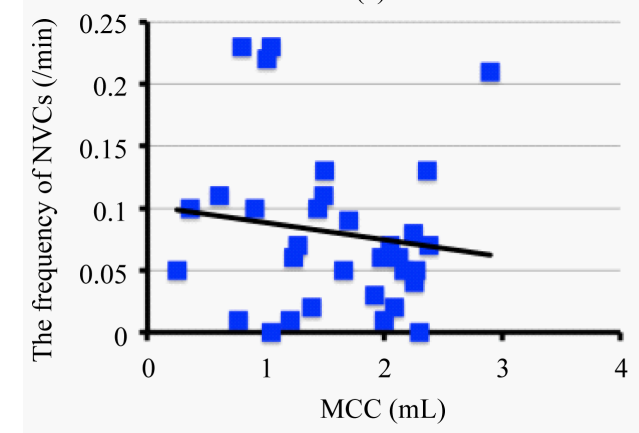

(c)

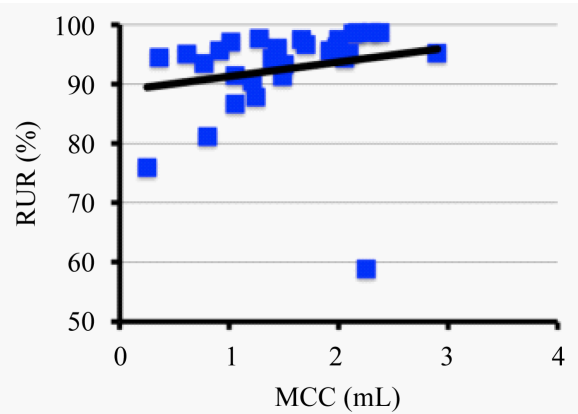

(b)

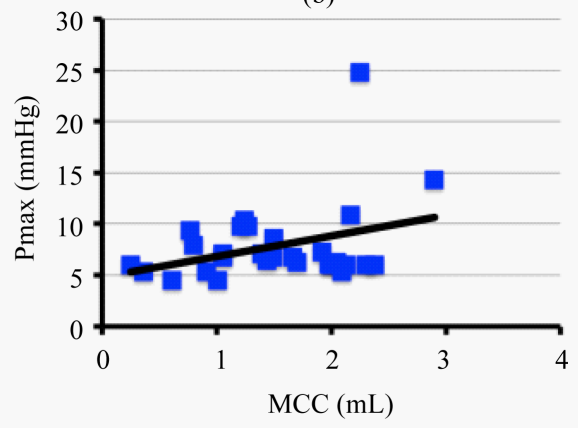

(d)

Figure 2. Scatter plots of maximum cystometric capacity (MCC) and each cystometric parameter with regression lines. MCC is significantly positively correlated to PVR (a) and RUR (b), but is not significantly correlated to the frequency of NVCs (c) and Pmax (d). NVCs, non-voiding contractions; PVR, postvoid residual urine volume; RUR, residual urine rate $(=\mathrm{PVR} \div(\mathrm{VV}+$ PVR) $\times 100$ ); Pmax, maximum bladder pressure during micturition.

and PVR in Group B was significantly smaller than in Group C (Table 2, Figure 3). RUR and Pmax in LCS rats were approximately $90 \%$ and $8 \mathrm{mmHg}$, respectively, regardless of group.

\subsection{MCC vs. NVCs}

The frequency of NVCs in LCS rats $(0.08 \pm 0.012 / \mathrm{min})$ was numerically higher, but not significantly different from that in sham rats $(0.04 \pm 0.011 / \mathrm{min})$. MCC was not significantly correlated to the frequency of NVCs (Table 1, Figure 2). In LCS rats, the frequency of NVCs did not significantly correlate to the CMG parameters (Table 1). The frequency of NVCs in each LCS group was not significantly different from that in sham-operated rats ( $\mathrm{p}=0.175$, Table 2). However, a Jonckheere-Terpstra trend test revealed a significant trend toward higher NVCs in the order of sham, Groups C, B, and A ( $p=0.036$, Figure 4). 
Table 2. Cystometric parameters in sham and groups A to C in LCS rats.

\begin{tabular}{ccccccc}
\hline Group & MCC $(\mathrm{mL})$ & NVCs $(/ \mathrm{min})$ & VV $(\mathrm{mL})$ & PVR $(\mathrm{mL})$ & RUR $(\%)$ & Pmax $(\mathrm{mmHg})$ \\
\hline Sham $(\mathrm{n}=10)$ & $1.34 \pm 0.23$ & $0.042 \pm 0.011$ & $1.56 \pm 0.25$ & $0.07 \pm 0.07$ & $4.50 \pm 0.02$ & $19.78 \pm 3.24$ \\
Group A $(\mathrm{n}=5)$ & $0.56 \pm 0.11^{* *+\pi}$ & $0.10 \pm 0.037$ & $0.062 \pm 0.09^{*}$ & $0.50 \pm 0.14^{* *+\pi}$ & $88.06 \pm 0.03^{*}$ & $6.62 \pm 1.69^{*}$ \\
Group B $(\mathrm{n}=13)$ & $1.30 \pm 0.07^{*}$ & $0.092 \pm 0.020$ & $0.088 \pm 0.05^{*}$ & $1.30 \pm 0.09^{* *}$ & $93.54 \pm 0.02^{*}$ & $7.36 \pm 1.05^{*}$ \\
Group C $(\mathrm{n}=14)$ & $2.22 \pm 0.07^{*}$ & $0.063 \pm 0.014$ & $0.141 \pm 0.06^{*}$ & $1.92 \pm 0.09^{*}$ & $93.41 \pm 0.02:$ & $9.00 \pm 1.14^{*}$ \\
\hline
\end{tabular}

mean \pm SEM. MCC, maximum cystometric capacity; VV, voided volume; PVR, postvoid residual urine volume; RUR, Residual urine rate $(=(\mathrm{PVR} \div(\mathrm{VV}+\mathrm{PVR})) \times 100)$; Pmax, maximum bladder pressure during micturition. ${ }^{*} \mathrm{p}<0.0001$ (vs. sham), ${ }^{\mathrm{p}}<0.0001$ (vs. Group C), "p $<$ 0.0001 (vs. Group B), Tukey-Kramer's HSD test.
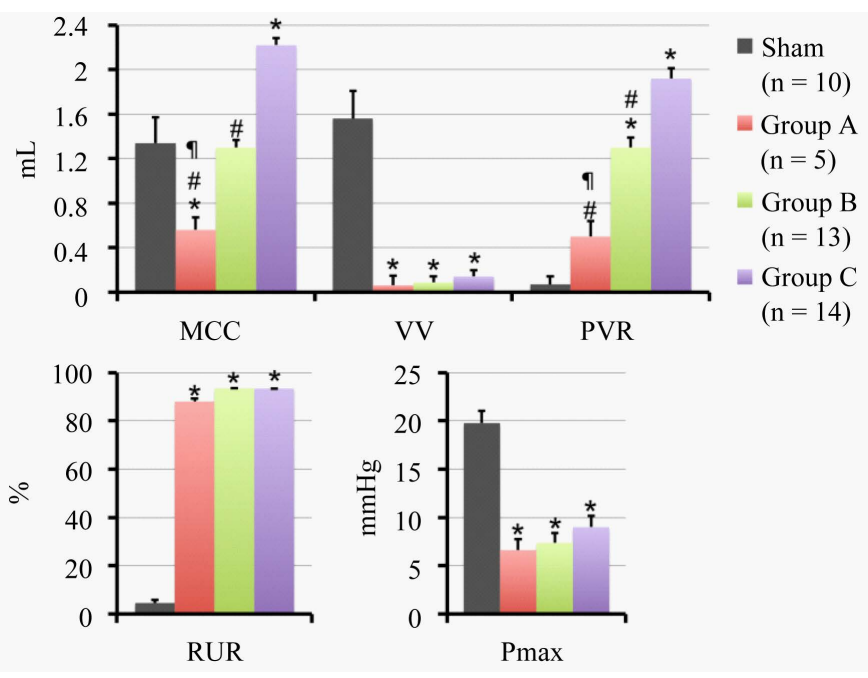

Figure 3. Cystometric parameters in sham and groups A to C in LCS rats. MCC, maximum cystometric capacity; VV, voided volume; PVR, postvoid residual urine volume; RUR, Residual urine rate $(=(P V R \div(\mathrm{VV}+\mathrm{PVR})) \times 100)$; Pmax, maximum bladder pressure during micturition. ${ }^{*} \mathrm{p}<0.0001$ (vs. sham), ${ }^{\#} \mathrm{p}<0.0001$ (vs. Group C), "p $<0.0001$ (vs. Group B), Tukey-Kramer's HSD test.

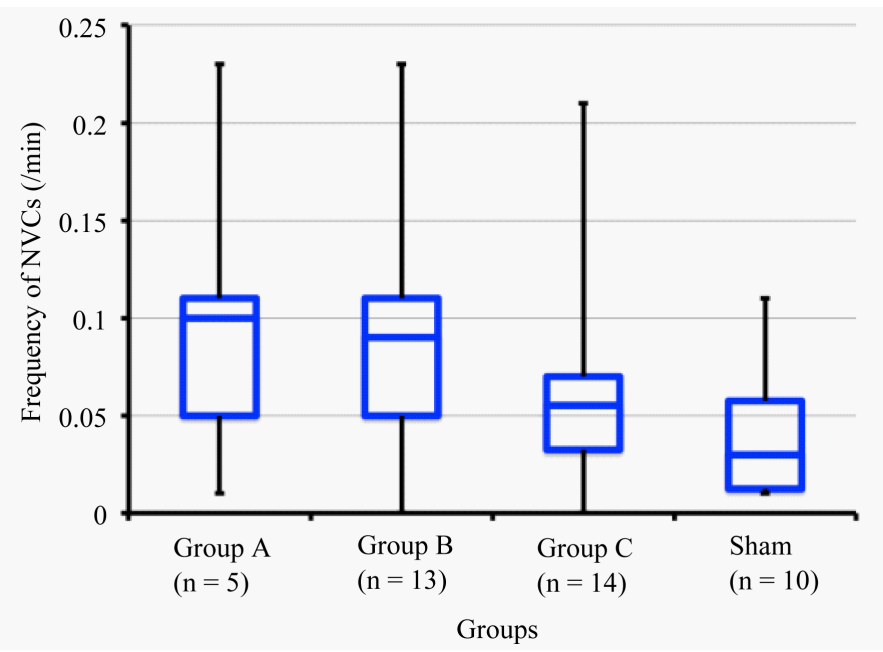

Figure 4. Frequency of non-voiding contractions (NVCs) in sham and LCS rats. A significant trend toward higher NVCs in the order of sham, Groups C, B, and A ( $\mathrm{p}=0.036$, Jonckheere-Terpstra trend test). 


\section{Discussion}

The concept of an "OAB to UAB transition” emerged from several basic and clinical studies [3]-[6] [12]. We recently developed a rat LCS model as a UAB model, and showed that the frequency of NVCs in LCS rats was numerically higher than that in sham rats [7]. We also found that MCC in LCS rats was not always significantly larger than that in sham rats. Therefore, in this study, we investigated whether voiding parameters in LCS rats without increased MCC would be better, and whether the frequency of NVCs would be increased in LCS rats with decreasing MCC. As a result, among LCS rats VV, RUR, and Pmax were not significantly different. On the other hand, PVR in Group A was significantly smaller than in Group B or C, and PVR in Group B was significantly smaller than in Group C. These results imply that the decreased PVR was probably due to the absence of increased MCC. In addition, UAB in LCS rats could be accompanied by increased frequency of NVCs, especially in rats with smaller MCC.

UAB has various etiologies, and infrasacral NB due to LCS seems to be one of the most common in aging populations [13]. Thirty to eighty percent of patients with LCS have lower urinary tract symptoms, mainly due to neurogenic UAB [14] [15]. That is why we have used the rat LCS model as a UAB model. We have reported lower urinary tract functions in the rat LCS model [7]. Compared with sham rats, we showed numerically higher frequency of NVCs in LCS rats and noticed that MCC in LCS rats was not always larger [7]. Recent studies noted that an $\mathrm{OAB}$ to $\mathrm{UAB}$ transition would be an important pathophysiology in the development of UAB [3]-[6]. In fact, results from experimental studies using diabetic or iliac atherosclerosis rat models provided some evidences of an OAB to UAB transition [2] [12]. Chancellor hypothesized that chronic muscular overactivity in $\mathrm{OAB}$ may lead to muscle fatigue or progressive ischemia, inflammation and oxidative damage caused by factors of OAB can lead to DU [3]. Because MCC in LCS rats did not always increase [7], we supposed that the $\mathrm{OAB}$ to UAB transition phenomenon would occur in the rat LCS model. If the OAB to UAB transition phenomenon is present in LCS rats and the phenomenon in LCS rats resembles those in diabetic or pelvic ischemic rat models [2]-[6], "transitional LCS rats" would not show increasing MCC, but would show increasing frequency of NVCs. Moreover, we suppose that voiding parameters would be better in LCS rats without increased MCC, and the frequency of NVCs would be increased with decreasing MCC. However, the present study showed that MCC did not correlate with voiding parameters except PVR and RUR. When LCS rats were divided into three groups based on the MCC \pm 2 SD of sham rats, the results were the same. Consequently, as shown in Table 2 and Figure 3, even LCS rats without increased MCC revealed the definitive features of DU.

The present study did not demonstrate the OAB to UAB transition that is seen in diabetic or atherosclerosis rat models [2] [12]. The LCS rat model was developed by cauda equina compression, which was thought to be so severe that all rats had significant DU after the rubber insertion. On the other hand, apart from spinal cord injury models or bilateral pelvic nerve transection models, the rat LCS model shows NVCs probably due to a partial decentralization [10]. In LCS rats, a decrease in local spinal blood flow was recognized [16]. It is conceivable that ischemic disturbances of the conus medullaris or irritation of the sacral roots, which were proposed as underlying mechanisms for development of DO in human LCS [9], play some role in the development of decreased or unchanged MCC. The resultant hyperactivity of the afferent pathway might prevent MCC from increasing. On the other hand, in the LCS rats, VV, PVR, RUR, and Pmax were consistently worse than in shamoperated rats, suggesting that efferent damage was sufficient to cause reproducible severe DU. Sone et al. reported that RUR in LCS patients was $48.3 \%$ and $54.5 \%$ in patients with DU and DO on cystometry, respectively [17]. However, PVR in DO patients $(131.7 \mathrm{~mL})$ was significantly smaller than that in DU patients $(227.0 \mathrm{~mL})$ because MCC in the former $(241.7 \mathrm{~mL})$ was significantly smaller than that in the latter ( $470.4 \mathrm{~mL})$. In cauda equina compression syndrome, $35 \%$ of DO patients also complained of urinary retention, while $56 \%$ of DU patients complained of that symptom [10]. The same paper showed that MCC in DO patients $(194.12 \mathrm{~mL})$ was significantly smaller than that in DU patients $(474.66 \mathrm{~mL})$. Podnar et al. reported that in cauda equina compression $70 \%$ of men and $85 \%$ of women had a normal cystometric pattern and $9 \%$ of men and $15 \%$ of women with a reduced MCC in the filling phase, but 59\% of men and $85 \%$ of women revealed an underactive or acontractile detrusor in the voiding phase [18]. Based on these clinical results, cauda equina compression causes significant voiding dysfunction even in DO patients, but does not frequently cause markedly increased MCC (such as more than $600 \mathrm{~mL}$ ) even in DU patients. Considering the fact that the cystometric findings of the rat LCS seem to be consistent with these clinical findings, more severe efferent dysfunction than the afferent dysfunction would be a cardinal feature in the rat LCS model. 
PVR in LCS rats was significantly correlated to MCC. Hyperactivity of the afferent pathway would dampen the increase in PVR despite poor voiding efficiency, although the present study does not provide definitive evidence that the OAB-like phenomenon is a compensatory mechanism for UAB. Recently, we reported lower urinary tract function in LCS rats after cholinesterase inhibitor (distigmine) administration [8]. Distigmine decreased PVR, but this decrease was not accompanied by increased Pmax or VV. Rather, decreased MCC was a possible contributing factor, and increased NVCs after administration of distigmine might contribute to decreased MCC. We are continuing further study of the lower urinary tract function of LCS rats after the administration of drugs other than distigmine.

It is reasonable to propose that the small size of the silicone rubber insert would allow the LCS rats to demonstrate the OAB to UAB transition phenomenon. In fact, we determined that this size and insertion method gives the rats the appropriate features of LCS: namely, no significant deficit in motor function under resting conditions, a reliable pattern of motor dysfunction after exercise, and identifiable detrusor contractions on awake CMG [7] [19]. Therefore, use of a different size of silicone rubber insert is not realistic in this model. To this end, however, the development of a model of gradually progressive cauda equina compression or constriction is needed in the future.

\section{Conclusion}

LCS rats showed DU to the same degree regardless of MCC. NVCs did not significantly increase in LCS rats with decreased MCC, but there was a significant trend toward higher NVCs with smaller MCC.

\section{References}

[1] Cucchi, A., Quaglini, S. and Rovereto, B. (2009) Proposal for a Urodynamic Redefinition of Detrusor Underactivity. Journal of Urology, 181, 225-229. http://dx.doi.org/10.1016/j.juro.2008.09.018

[2] Nomiya, M., Yamaguchi, O., Akaihata, H., Hata, J., Sawada, N., Kojima, Y. and Anderson, K.E. (2014) Progressive Vascular Damage May Lead to Bladder Underactivity in Rats. Journal of Urology, 191, 1462-1469. http://dx.doi.org/10.1016/j.juro.2013.10.097

[3] Chancellor, M.B. (2014) The Overactive Bladder Progression to Underactive Bladder Hypothesis. International Urology and Nephrology, 46, S23-S27. http://dx.doi.org/10.1007/s11255-014-0778-y

[4] Yoshida, M. and Yamaguchi, O. (2014) Detrusor Underactivity: The Current Concept of the Pathophysiology. LUTS, 6, 131-137. http://dx.doi.org/10.1111/luts.12070

[5] Andersson, K.E., Nomiya, M., Sawada, N. and Yamaguchi, O. (2014) Pharmacological Treatment of Chronic Pelvic Ischemia. Therapeutic Advances in Urology, 6, 105-114. http://dx.doi.org/10.1177/1756287214526768

[6] Yamaguchi, O., Nomiya, M. and Andersson, K.E. (2014) Functional Consequences of Chronic Bladder Ischemia. Neurourology and Urodynamics, 33, 54-58. http://dx.doi.org/10.1002/nau.22517

[7] Sekido, N., Jyoraku, A., Okada, H., Wakamatsu, D., Matsuya, H. and Nishiyama, H. (2012) A Novel Animal Model of Underactive Bladder: Analysis of Lower Urinary Tract Function in a Rat Lumbar Canal Stenosis Model. Neurourology and Urodynamics, 31, 1190-1196. http://dx.doi.org/10.1002/nau.21255

[8] Sekido, N., Kida, J., Wakamatsu, D., Okada, H. and Matsuya, H. (2014) Effects of a1 Antagonist and Cholinesterase Inhibitor on Cystometric Parameters in Lumbar Canal Stenosis Rats with Underactive Bladder. Urology, 84, 1248.e91248.e15. http://dx.doi.org/10.1016/j.urology.2014.07.036

[9] Yamanishi, T., Yasuda, K., Sakakibara, R., Murayama, N., Hattori, T. and Ito, H. (1998) Detrusor Overactivity and Penile Erection in Patients with Lower Lumbar Spine Lesions. European Urology, 34, 360-364. http://dx.doi.org/10.1159/000019756

[10] Kim, S.Y., Kwon, H.C. and Hyun, J.K. (2014) Detrusor Overactivity in Patients with Caudaequina Syndrome. Spine, 39, E955-E961. http://dx.doi.org/10.1097/BRS.0000000000000410

[11] Nishiguchi, J., Kwon, D.D., Kaiho, Y., Chancellor, M.B., Kumon, H., Snyder, P.B. and Yoshimura, N. (2006) Suppression of Detrusor overactivity in Rats with Bladder Outlet Obstruction by a Type 4 Phosphodiesterase Inhibitor. BJU International, 99, 680-668. http://dx.doi.org/10.1111/j.1464-410X.2006.06643.x

[12] Daneshgari, F., Liu, G., Birder, L., Hanna-Mitchell, A.T. and Chacko, S. (2009) Diabetic Bladder Dysfunction: Current Translational Knowledge. The Journal of Urology, 182, S18-S26. http://dx.doi.org/10.1016/j.juro.2009.08.070

[13] Kalichman, L., Cole, R., Kim, D.H., Li, L., Suri, P., Guermazi, A. and Hunter, D.J. (2009) Spinal Stenosis Prevalence and Association with Symptoms: The Framingham Study. The Spine Journal, 9, 545-550.

http://dx.doi.org/10.1016/j.spinee.2009.03.005 
[14] Perner, A., Andersen, J.T. and Juhler, M. (1997) Lower Urinary Tract Symptoms in Lumbar Root Compression Syndromes: A Prospective Study. Spine, 22, 2693-2697. http://dx.doi.org/10.1097/00007632-199711150-00019

[15] Tsai, C.H., Chou, E.C.L., Chou, L.W., Chen, Y.J., Chang, C.H., Tsou, H.K. and Chen, H.T. (2010) The Evaluation of Bladder Symptoms in Patients with Lumbar Compression Disorders Who Have Undergone Decompressive Surgery. Spine, 35, E849-E854. http://dx.doi.org/10.1097/BRS.0b013e3181d55ad8

[16] Takenobu, Y., Katsube, N., Marsala, M. and Kondo, K. (2001) Model of Neuropathic Intermittent Claudication in the Rat: Methodology and Application. Journal of Neuroscience Methods, 104, 191-198. http://dx.doi.org/10.1016/S0165-0270(00)00342-3

[17] Sone, A., Moda, Y., Koyama, K. and Tanaka, H. (1994) Voiding Dysfunctions in Patients with Lumbar Canal Stenosis. Nippon Hinyokika Gakkai Zasshi, 85, 611-615.

[18] Podnar, S., Trsinar, B. and Vodusek, D.B. (2006) Bladder Dysfunction in Patients with Caudaequina Lesions. Neurourology and Urodynamics, 25, 23-31. http://dx.doi.org/10.1002/nau.20188

[19] Nakai, K., Takenobu, Y., Eguchi, K., Takimizu, H., Honjo, K., Akimaru, S., Maegawa, H., Marsala, M. and Katsube, N. (2002) The Effects of OP-1206 $\alpha$-CD on Walking Dysfunction in the Rat Neuropathic Intermittent Claudication Model. Anesthesia Analgesia, 94, 1537-1541. http://dx.doi.org/10.1213/00000539-200206000-00030 